\title{
Brechó-SocialSECO: Uma Ferramenta para Apoiar Modelagem e Análise de Ecossistemas de Software
}

\author{
Gabriel Barbosa, Thaiana Lima, Rodrigo Pereira dos Santos, Cláudia Werner \\ COPPE/UFRJ - Universidade Federal do Rio de Janeiro \\ Caixa Postal 68511 - CEP 21941-972 - Rio de janeiro, RJ, Brasil \\ \{gabrielsb, thaiana, rps, werner\}acos.ufrj.br
}

\begin{abstract}
In any software system, there is interaction between components. By joining a technical network of artifacts with a social environment, a sociotechnical network can be created. In order to develop a tool to model, analyze and encourage social relations in this socio-technical network, Brechó SocialSECO is proposed. It extends a component library called Brechó. The goal is to provide a better understanding of software ecosystem and a support for decision-making. This paper presents the tool and its operation.
\end{abstract}

Resumo. Em todo sistema de software, existe interação entre componentes. Ao unir uma rede técnica de artefatos com um ambiente social, cria-se uma rede socio-técnica. Visando desenvolver uma ferramenta que modele, analise e incentive as relações sociais na rede socio-técnica, foi proposta a Brechó SocialSECO. Estendeu-se a biblioteca de componente Brechó para melhor entender ecossistemas de software e dar suporte para tomadas de decisão. Este trabalho apresenta a ferramenta e seu funcionamento.

\section{Introdução}

Mecanismos de socialização são meios para estimular a relação entre atores de um sistema. Segundo Seichter et al. (2010), a comunicação e a interação entre esses atores durante o processo de desenvolvimento de software são realizadas por meio dos artefatos que eles compartilham. Jansen et al. (2009) definem um Ecossistema de Software (ECOS) como um conjunto de atores funcionando como uma unidade que interage com um mercado distribuido entre software e serviços, juntamente com as relações entre estas entidades. Essas relações são apoiadas por uma plataforma tecnológica ou por um mercado comum, e realizadas pela troca de informação, recursos e artefatos. Nesse contexto, a intensidade da interação entre atores e artefatos no ECOS leva à fusão da rede de atores (rede social) e da rede de artefato (rede técnica), resultando em uma rede socio-técnica (Cukierman et al., 2007).

A aplicação de conceitos da análise de interações sociais aparece em poucos trabalhos de ECOS, de modo que seu domínio ainda carece de efetiva exploração desses conceitos (Santos \& Oliveira, 2013). À medida que uma organização evolui, deixando de construir um produto de software isolado, e busca parcerias para abrir seus negócios, ela ultrapassa suas fronteiras e encontra um ecossistema formado por várias outras (Berk et al., 2010). Cria-se então a necessidade de se estudar ECOS como um conjunto formado por plataforma, rede de atores (stakeholders) e rede de artefatos (Manikas \& Hansen, 2013). A partir disto, surge outra necessidade: gerir as informações provenientes deste ECOS. Com o aumento das interações entre os atores e artefatos, 
uma gama de informações novas surge a todo momento no ECOS, impactando o seu estado e a organização de seus elementos (Lima et al., 2014).

Em Peréz et al.(2012), é apresentada a ferramenta SECONDA, que visa a análise do ECOS e sua evolução. Por meio de métricas de projeto, analisa-se o comportamento do ECOS e suas principais características. Porém, a ferramenta apresenta limitações, pois não contempla as relações sociais que ocorrem durante o ciclo de vida do ECOS. Outra carência é a previsão de tendências, pois a ferramenta analisa informações coletadas e não prevê necessidades e tendências sobre as relações no ECOS. O Portal do Software Público Brasileiro ${ }^{1}$, por sua vez, é uma plataforma de ECOS que possui uma gama de interações sociais, como fóruns e comunidades. Além de um repositório para usuários finais e desenvolvedores, o SPB armazena informações das relações entre atores. Porém, o SPB não possui gamificação, mineração de dados e a visualização da rede, ferramentas que possuem que fazem parte da proposta deste trabalho. Outra ferramenta é apresentada por Goeminne et al. (2010), constituída por um framework que capta informações do ECOS por meio de extensões e, ao utilizar mineração de dados, gera um conjunto de gráficos e dados sobre o ECOS e seu ciclo de vida. O framework, porém, por ser bastante genérico, necessita de um conjunto de implementações para se adequar a cada ECOS, necessitando programar as informações que se deseja minerar.

O objetivo deste trabalho é apresentar a Brechó-SocialSECO, uma ferramenta para incentivar relações sociais no ECOS e transformar os dados gerados dessas interações em informações úteis para a tomada de decisão dos stakeholders, ajudando na compreensão das demandas e necessidades que surgem da rede. Para isso, a ferramenta apoia na modelagem e análise de ECOS. Com o suporte de mecanismos de análise e mineração implementados na Brechó-SocialSECO, um plugin de visualização da rede socio-técnica do ECOS foi desenvolvido na ferramenta Gephi ${ }^{2}$. São obtidas informações acerca da formação de grupos com interesses similares e a identificação de tendências do mercado, entre outras análises. Além desta seção de introdução e fundamentação, a Seção 2 apresenta a ferramenta Brechó-SocialSECO, com a sua arquitetura, mecanismos e implementação como extensão da biblioteca de componentes e serviços Brechó; e a Seção 3 conclui o artigo com algumas considerações finais e trabalhos futuros.

\section{Brechó-SocialSECO}

Repositórios e bibliotecas de componentes e serviços são importantes para alcançar objetivos de Reutilização de Software, servindo como vitrine para buscar e recuperar componentes existentes na organização e prontos para a reutilização (Overhage \& Thomas, 2004). Segundo Pujatti et al. (1994), a reutilização diminui os esforços de desenvolvimento e manutenção dos projetos de software. Um dos protótipos disponíveis nessa área é a biblioteca Brechó $^{3}$, que permite o desenvolvimento de extensões. A Brechó é um sistema de informação Web com uma base de aplicações, componentes, serviços, produtores/consumidores, com mecanismos de armazenamento, documentação, publicação, busca e recuperação (Werner et al., 2009).

\footnotetext{
${ }^{1}$ Disponível em: www.softwarepublico.gov.br/

${ }^{2}$ Disponível em: http://gephi.github.io

${ }^{3}$ Disponível em: http://reuse.cos.ufrj.br/brecho
} 
A fim de lidar com um conjunto de informações relativas ao funcionamento do ECOS e incentivar as relações sociais, a Brechó-SocialSECO é proposta como um ferramental de apoio. A Brechó-SocialSECO estende a Brechó ${ }^{4}$ para enriquecê-la com mecanismos sociais que apoiem a manutenção de redes socio-técnicas (Lima et al., 2014). A solução apresenta uma arquitetura centrada na biblioteca Brechó, com a inserção dos mecanismos sociais e de mineração, discutidos respectivamente nas Subseções 2.1 e 2.2. Os mecanismos sociais são introduzidos na biblioteca para apoiar a geração de dados, cujas informações extraídas resultam em gráficos gerados pelo plugin da ferramenta Gephi ${ }^{5}$, por meio do refinamento realizado por mecanismos de mineração de dados. A Figura 1 mostra a arquitetura da solução.

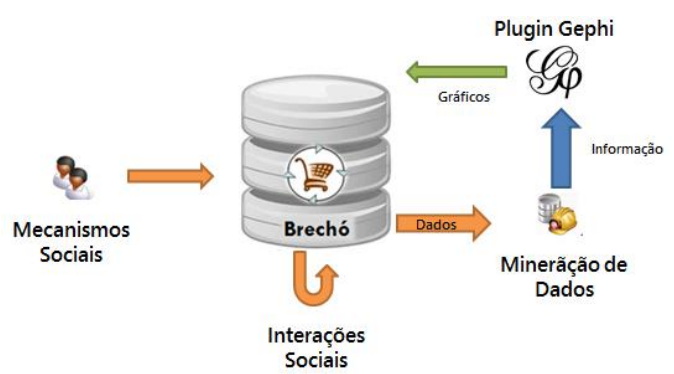

Figura 1. Arquitetura da Solução

\subsection{Mecanismos Sociais}

Os mecanismos sociais inseridos na biblioteca por este trabalho foram: equipes, fórum, perfil dos usuários, sistemas de pontuação e de sugestão, e notícias. Para que o usuário tenha acesso a um portal voltado para o uso dos mecanismos sociais de ECOS implantados, foi incorporada a página "Minha Rede” na Brechó (Figura 2).

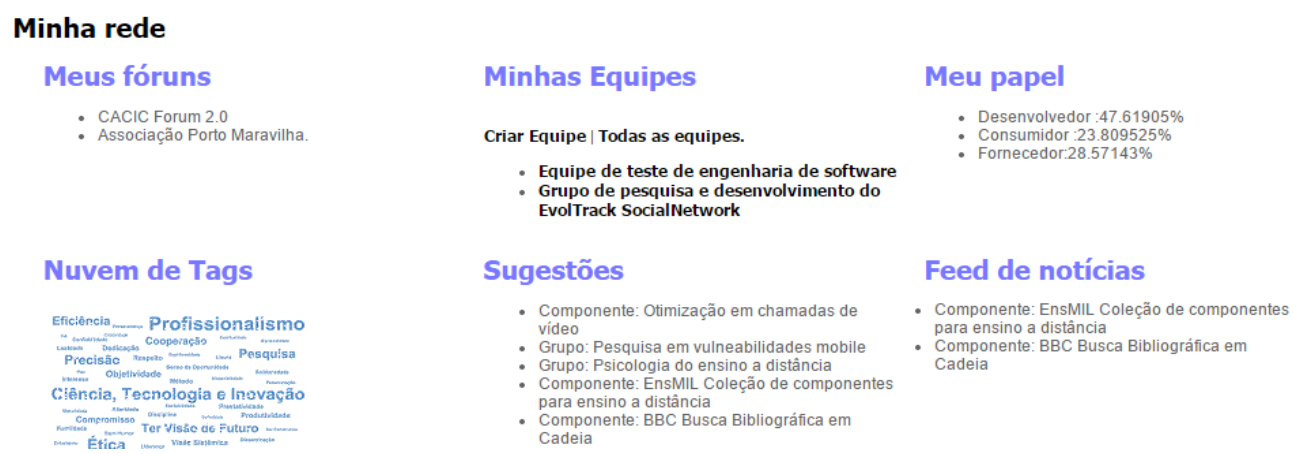

Figura 2. Tela "Minha Rede" na Brechó-SocialSECO

Como a biblioteca de componentes passa a ser apoiada nas relações sociais e decisões conjuntas dos usuários, a formação de equipes de desenvolvimento tende a ser algo comum (Seichter et al., 2010). Procurando satisfazer esta condição, foi proposta a criação de um novo tipo de usuário, denominado Equipe. A Equipe é composta por um conjunto de outros usuários em que, por padrão, o usuário que criou a equipe é o "administrador" da conta em questão. O administrador da equipe pode delegar

\footnotetext{
${ }^{4}$ Esta biblioteca foi escolhida por utilizar desenvolvimento baseado em componentes (Szyperski et al., 2002), facilitando a manutenção e extensão (reutilização), e pelo acesso ao código-fonte.

5 Esta ferramenta foi escolhida por ser plataforma open source que disponibiliza API para desenvolvimento, construída na linguagem Java e tendo como input uma conexão com banco de dados.
} 
privilégios a cada usuário (e.g., comprar componentes, criar tópicos em fóruns ou publicar novos componentes na biblioteca). Um usuário pode participar de mais de uma equipe. Como a compra é feita por licenças, uma equipe pode comprar um conjunto de licenças para todos os membros da equipe, ou apenas poucas licenças para alguns deles.

Cada equipe conta com um fórum, que serve como recurso para a troca de informações. $\mathrm{O}$ fórum é um repositório de mensagens separadas por tema ou assunto, sendo constituído por três setores: Tópicos, Sugestões e Requisitos. Os tópicos são constituídos por discussões que, por sua vez, são formadas por mensagens. A abertura de discussões e mensagens é livre. Cada mensagem apresenta duas opções para leitores: Positivo e Negativo. Quanto mais bem cotada for uma mensagem (i.e., mais escolhas/avaliações positivas), mais útil e relevante é considerada para a comunidade. A avaliação (positivo/negativo) é feita pelos próprios usuários do fórum.

O mecanismo de sugestões é formado por mensagens vindas dos usuários do componente. Visa criar um canal de comunicação entre usuários e desenvolvedores. $\mathrm{Na}$ Brechó-SocialSECO, demandas e necessidades do ECOS (e.g., novas funcionalidades ou componentes) podem ser compartilhadas como requisitos. Quando um novo requisito é adicionado, é possível que um usuário ou equipe sinalize interesse em solucionar este requisito. $\mathrm{O}$ administrador do fórum pode permitir que esses usuários ou equipes desenvolvam uma solução de software oficial, ou informar que uma equipe particular já está solucionando o requisito. A tela do fórum enriquecida com o mecanismo de sugestão (aba "requisitos") pode ser vista na Figura 3.

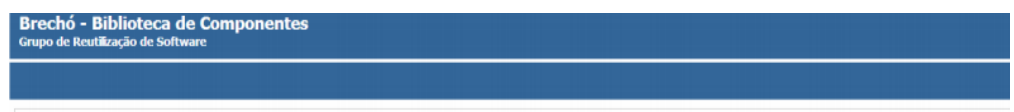

Componente ABC $>$ Fórum > Discussões
\begin{tabular}{|l|l|l|l|}
\hline Tópicos & Tema & Posts & Último Post \\
\hline Sugestões & Discussão 1 & 29 & $10 / 02 / 2014$ 20:30 \\
\hline Requisitos & Discussão 2 & 32 & $01 / 01 / 2014$ 02:15 \\
\hline & Discussão 3 & 206 & $16 / 02 / 201415: 23$ \\
\cline { 2 - 5 } & \multicolumn{3}{|l}{} \\
\hline
\end{tabular}

Figura 3. Tela do mecanismo "Fórum" na Brechó-SocialSECO

A versão original da Brechó possuía um sistema de pontos de recompensa implementado, que incentiva o ator a dar feedback de um componente adquirido a um produtor. Porém, esse sistema não contemplava a área social, o que motivou a sua expansão para criar um mecanismo que impulsionasse o crescimento do ECOS e a obtenção de seus dados, incentivando o ator a contribuir com informações sobre os componentes por meio do fórum. Ao acessar o fórum do componente adquirido, o usuário pode manifestar interesse em solucionar um requisito em aberto. Após criar e publicar essa solução na Brechó, é analisado o seu impacto no ECOS do componente. Essa analise é feita de diversas maneiras, e.g., número de acessos, número de downloads, "likes" da solução, entre outros.

As recompensas em pontos são dadas ao autor da solução, conforme a sua utilidade para a comunidade, determinada pela avaliação dos próprios membros. Como usuários ativos recebem mais pontos de recompensa, desenvolvedores e empresas visam este tipo de usuário em seus ecossistemas, estimulando o desenvolvimento do componente e toda a comunidade em sua volta (Manikas \& Hansen, 2013). A Brechó 
identifica os atores em três principais papéis: Produtor, Consumidor e Gerente da Biblioteca (Werner et al., 2009). Com o aumento das interações entre os atores dentro de um ECOS, foi preciso aumentar a precisão da identificação dos atores na Brechó, de forma a entender melhor como cada ator se situa dentre esses três papéis principais. Para isso, foi preciso atribuir pontuações para cada ação do ator, de modo que cada ação seja ponderada pelo grau associado a cada um dos três papéis principais. Portanto, há um leque de possibilidades, já que os atores podem exercer papeis mesclados (i.e., participar de diferentes formas), com diferentes graus de atribuição (e.g., 20\% do tipo A, 30\% do tipo B e $50 \%$ do tipo C). Por fim, um feed de notícias com as últimas ações realizadas nos mecanismos sociais, na tela "Minha Rede", está em implementação.

\subsection{Mecanismos de Mineração}

Para que as informações obtidas das relações sociais no ECOS sejam transformadas em informações úteis para os stakeholders, é necessário efetuar a mineração dos dados. Este trabalho foi baseado em dois mecanismos de mineração de dados: a mineração de texto (utilizando mensagens de Forúns) e análise de associação (associação de componentes de atores com perfis similares). Após a mineração de dados, os resultados são passados para um ferramental de exibição de informação (neste trabalho, é utilizado o Gephi).

A mineração de texto tem por objetivo descobrir tendências de assuntos presentes no fórum de discussão. Assim, é possível criar conteúdos personalizados para cada tipo de fórum ou assunto discutido. Um desses conteúdos é a nuvem de tags, que é constituída por um conjunto de palavras ou termos regularmente comentados ou discutidos em um fórum, e que se apresentam visualmente e proporcionalmente à sua ocorrência nos tópicos. Assim, assuntos bem cotados e que apresentem palavras-chave como "urgência", "necessidade" e "sugestão" podem ser identificados pela ferramenta como possíveis sugestões, alertando o desenvolvedor/produtor do componente de uma tendência do mercado, além de alimentar o sistema de sugestão.

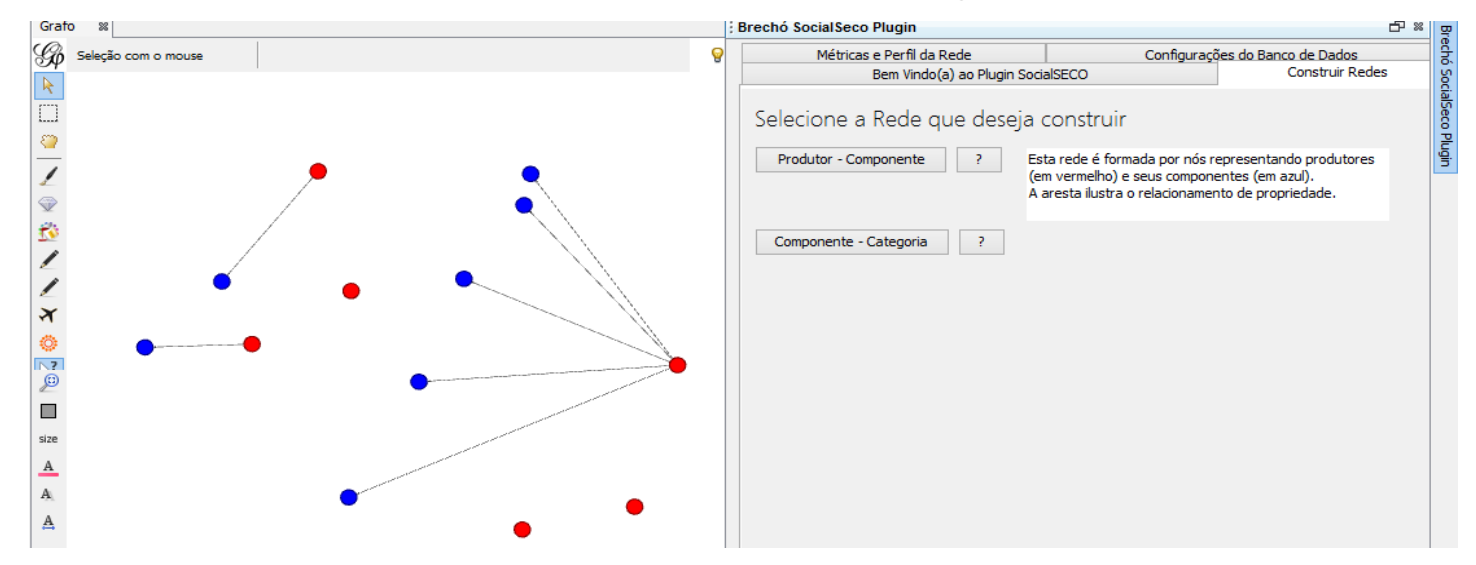

Figura 4. Tela para análise da rede sócio-técnica no plug-in do Gephi

Baseado nas relações entre os atores, a análise de associação procura associar componentes adquiridos por dois ou mais atores. Busca-se encontrar componentes afastados que acabam sendo relacionados, seja pela complementação de suas funções ou por suas dependências. A maior parte dessa informação é retirada dos componentes adquiridos pelos atores do ECOS. Por exemplo, um ator possui um grupo de componentes e, caso haja um ator com perfil similar, é possível que ele tenha interesse 
no mesmo grupo de componentes, sendo possível recomendar componentes para atores de mesmo perfil. Para apoiar nesta tarefa, a Figura 4 mostra um diagrama de rede sociotécnica exibido pelo plugin construído, que permite realizar uma análise da rede.

\section{Conclusão}

Este trabalho apresentou a Brechó SocialSECO, ferramenta que implementa mecanismos sociais e de mineração, estendendo a biblioteca Brechó ao inserir uma visão social para apoiar modelagem e análise de ECOS. Baseados nas relações sociais, dados destas interações transformam-se em novas informações, por meio de técnicas de mineração de dados, ajudando na tomada de decisões e expansão do ECOS. Como trabalhos futuros, um sistema de clustering, que traga informações sobre grupos de perfis semelhantes de usuário, e um sistema de sugestão baseado nessas informações, estão sendo implementados na solução. Além disso, um feed de notícias que informe as alterações realizadas nos mecanismos sociais no repositório está em implementação. Por fim, a inserção de uma análise temporal dos dados coletados e dos gráficos gerados, permitindo que diferentes stakeholders possam analisar o ciclo de vida do ECOS e as mudanças dos perfis de seus usuários, serão implementados.

\section{Referências}

Berk, I., Jansen, S., Luinenburg L. (2010) "Software Ecosystems: A Software Ecosystem Strategy Assessment Model”. In: 4th ECSA, IWSECO, Copenhagen, Denmark, 135-142.

Cukierman, H.L., Teixeira, C., Prikladnicki, R. (2007) "Um Olhar Sociotécnico sobre a Engenharia de Software”. Revista de Informática Teórica e Aplicada 14(2):207-227.

Goeminne, M. et al. "A Framework for Analysing and Visualising Open Source Software Ecosystems". In: Joint ERCIM EVOL/IWPSE, Antwerp, Belgium, 42-47.

Jansen, S., Finkelstein, A., Brinkkemper, S. (2009) "A Sense of Community: A research Agenda for Software Ecosystems". In: 31st ICSE, Vancouver, Canada, 187-190.

Lima, T. et al. (2014) "Uma Abordagem Socio-técnica para Apoiar Ecossistemas de Software". iSys: Revista Brasileira de Sistemas de Informação 7(3):19-37.

Manikas, K., Hansen, K.M. (2013) "Software Ecosystems - A Systematic Literature Review". The Journal of Systems and Software 86(5):1294-1306.

Overhage, S., Thomas, P. (2004) "A Business Perspective on Component Trading: Criteria, Immaturities, and Critical Success Factors". In: 30th Euromicro, Rennes, France, 108-117.

Pérez, J. et al. (2012) "SECONDA: Software Ecosystem Analysis Dashboard". In: 16th CSMR, Szeged, Hungary, 527- 530.

Pujatti, L. et al. (1994) “Ambiente de Reutilização de Software: Classificação de Módulos em Tecnologia de Composição". In: II Jornadas da USP, SUCESU-SP. São Paulo, Brasil, 46-57.

Santos, R., Oliveira, J. (2013) "Análise e Aplicações de Redes Sociais em Ecossistemas de Software”. In: IX SBSI, João Pessoa, Brasil, v. 2, 19-24.

Szyperski, C., Gruntz, D., Murer, S. (2002) "Component Software - Beyond Object-Oriented Programming". New York: Addison-Wesley and ACM Press, 2. ed.

Seichter, D. et al. (2010) "Knowledge Management in Software Ecosystems: Software Artefacts as First-class Citizens". In: 4th ECSA, IWSECO, Copenhagen, Denmark, 119-126.

Werner, C. et al. (2009) "Towards a Component and Service Marketplace with Brechó Library”. In: IADIS International Conference WWW/Internet 2009, Rome, Italy, 567-574. 